\title{
Subthreshold psychiatry
}

\section{Ahmed Okasha}

Director, WHO Collaborating Center for Research and Training in Mental Health, Okasha Institute of Psychiatry, Ain Shams University, Egypt, email aokasha@internetegypt.com

A re we in need of including in our diagnostic systems a new category of subthreshold psychiatry? Studies have shown that we are faced in our daily clinical practice with many patients who do not fulfil the criteria of either ICD-10 or DSM-IV (at best they may be included under 'atypical', 'unspecified' or 'not elsewhere classified'). Subthreshold cases or prodromal psychotic or non-psychotic clinical cases are encountered frequently in clinical practice, especially primary care (Knappe et al, 2008), but because of some ethical and nosological issues their needs are unmet. Pharmacological interventions for such conditions are denied in some countries, especially with managed care, where maximisation of profit and minimisation of cost are often the main objectives. It has been reported that the early treatment of many disorders ensures a better outcome and better assimilation in society and reduces residual manifestations of disease. Recent data suggest that the impairment and disability caused by subsyndromal disorders are almost equal to those caused by syndromal ones. We need more scientific data and research studies to evaluate the course, outcome and value of treating such disorders.

Early identification of the prodromal phase of a psychotic illness can lead to earlier treatment and perhaps prevention of a first psychotic episode. Up to $35 \%$ of 12 - to 30 -year-old individuals with a family history of psychosis plus a recent deterioration in functioning or new subsyndromal psychotic symptoms become psychotic within 2-3 years, which suggests that these factors have important diagnostic implications (Cadenhead et al, 2010). It was found in the $A B C$ Schizophrenia Study that in three-quarters of the cases initial psychotic symptoms were preceded by a prodromal phase of several years' standing, which began with non-specific signs such as negative and depressive symptoms (subthreshold) (Häfner et al, 1998). These had had adverse effects (including social disabilities) on those who subsequently developed schizophrenia (Löffler \& Häfner, 2000). To adhere only to criteria specifying a number of symptoms or their duration could be misleading and we should take into consideration the level of disability and impairment.

\section{Morbidity and prevalence}

In post-traumatic stress disorder (PTSD), no significant differences were found between patients with full PTSD and those with subthreshold PTSD regarding their degree of impairment. In fact, though, it is rather difficult to identify clinical criteria that delineate between full-blown PTSD and subthreshold PTSD (Zlotnik et al, 2002).
Subthreshold symptoms in bipolar disorder impair functioning and diminish quality of life. In one study, a reassessment of bipolar-spectrum disorders with the inclusion of patients with subthreshold symptoms revealed a prevalence at least fivefold greater than found with traditionally defined syndromal diagnostic criteria (Berk et al, 2008). The authors said that appropriate therapeutic interventions for the subthreshold manifestations should be considered even when threshold-level symptoms are absent.

In a 20-year follow-up study, patients with type I and type II bipolar disorder were found to experience subthreshold affective symptoms for half their lives (Judd et al, 2005). Many healthy people report depressive and hypomanic symptoms and many are identifiable as manifesting depressive, hypomanic and cyclothymic temperaments, which appear to predispose to the respective affective disorders and personality disorders. Only about 15\% of the population report no such symptoms over their lifetime and are 'supernormal', with very low scores for vegetative lability and neuroticism (Merikangas et al, 2007).

Studies have demonstrated that depressive symptoms in various combinations, including subthreshold conditions, are present in as much as $24 \%$ of the population. This suggests that subthreshold and full syndromic disorders can be considered as falling along a spectrum, with subthreshold disorders being viewed as quantitatively milder than, but qualitatively similar to, full syndromic disorders (Shankman et al, 2008).

Karsten et al (2010) suggested that subthreshold depression based on functional impairment is better defined by symptom severity than by number of DSM-IV symptoms.

\section{Types of subthreshold disorder}

The only specific subthreshold diagnosis in either ICD-10 or DSM-IV is 'mixed anxiety depression disorder' (MADD), presented in ICD-10 under F41.2, 'Other anxiety disorders'. Data from the National Psychiatric Morbidity Survey suggest that MADD may account for half of all cases of common mental disorders in Britain. Of those with MADD, 12\% reported a lifetime suicide attempt and $20 \%$ of all disability days in Britain occurred in people with MADD, accounting for around half of all the disability days occurring in people with common mental disorders (Das-Munshi et al, 2008).

There is a growing literature on subthreshold anxiety disorders, substance use disorders, conduct disorder, antisocial personality disorder and eating disorder. A family study of a variety of subthreshold disorders (MADD, bipolar disorder, anxiety disorders, alcohol use disorders, drug use disorders, 
and conduct disorder/antisocial personality disorder) in a large community sample of young adults found that a subthreshold disorder may be associated with multiple full syndromic disorders and vice versa. Just as there is high comorbidity between full syndromic disorders, there is also high comorbidity between subthreshold conditions. A study on social anxiety disorder (SAD) above and below the diagnostic threshold found that the 12-month prevalence rate for above-threshold SAD was $2 \%$, while it was 3\% for subthreshold and symptomatic social anxiety (Lewinsohn et al, 2004).

Subthreshold eating disorders have been frequently observed in transcultural studies. In a study of 371 Egyptian adolescent girls (age range 13-18 years; $4.4 \%$ of the target population), only two cases met the ICD-10 research criteria for atypical anorexia nervosa (0.5\% of the sample), while 26 cases met the criteria for atypical bulimia nervosa (7.0\%) and 32 cases the criteria for unspecified eating disorders (8.6\%). The author suggested that a culture-sensitive nosology for eating disorders was needed (Mahmoud, 1997).

\section{Subthreshold personality disorder}

Perhaps the most challenging field of research on cultural issues beyond the questions of boundaries between normality and abnormality is in personality functions. Cultural factors can contribute to the debate on whether personality disorders should or should not be considered autonomous mental disorders, or whether some of them are best considered as variants of Axis I disorders (e.g. schizotypal personality disorder as a variant of schizophrenia and borderline personality disorder as a variant of bipolar disorder).

The reliability and validity of the current diagnostic criteria of personality disorders in both ICD-10 and DSM-IV are low when applied in the Arab and North African regions. The schizoid, obsessive and avoidant personality disorders can have religious connotations, for example, and may even be desirable traits. It may be particularly problematic cross-culturally to apply categorical criteria developed in one culture to members of other cultures, for whom relevant categorical thresholds may differ. Again, religious perspectives in traditional societies may offer alternative interpretations of personality disorders: schizotypal features could connote closeness to God, schizoid features a kind person, paranoid features carefulness; similarly, avoidant behaviour may be praised by some religious conservatives who believe that mixing the sexes is unacceptable and who cherish shyness and avoidance, and anankastic traits may be perceived as meticulousness in following religious rituals. Culture thus affects the perception of mental symptoms - of what is a mental disorder and what is an accepted social trait (e.g. in traditional and religious societies negative symptoms may connote deeper contemplation of God, while positive symptoms may be attributed as a gift from God for extraordinary perception).

\section{Need for dimensional measures}

The addition of continuous, 'dimensional' measures to the various diagnostic domains might help to resolve some of the critical taxonomic issues currently facing the field of mental health. Regier et al (2009) recognised that categorical and dimensional approaches to diagnosis are important both for clinical work and for research, and that the ideal taxonomy would offer both. To avoid diagnostic chaos, the dimensional scale must reflect the categorical definition and the two must have an obvious relationship to one another.

It is clear that the inclusion of subthreshold or subsyndromal psychiatric disorder should have a place in our current nosology. The suffering, morbidity and functional impairment are almost equal to those of full syndromal disorders. The inclusion of those disorders not accommodated in the current nosology, whether ICD-10 or DSM-IV-TR, should be attempted in the proposed categorical dimensional system in ICD-11 and DSM-V so that we can ameliorate the suffering of people with these neglected mental disorders (Okasha, 2008).

There are, though, four ethical questions to be answered:

o Should we treat people who do not have a disorder by the current criteria of international classifications?

O Should we take 'quality of life' and 'disability' more into consideration in our diagnostic system?

O Should we include subthreshold disorders as part of the dimensional spectrum, rather than adding them as separate disorders?

O If we expand the scope for intervention, how can we address the risk of inappropriate medicalisation and iatrogenic harm from overdiagnosis and balance this against amelioration of suffering and disability caused by subthreshold disorders?

\section{References}

Berk, M., Ng, F., Wang, W., et al (2008) The empirical redefinition of the psychometric criteria for remission in bipolar disorder. Journal of Affective Disorders, 106, 153-158.

Cadenhead, K. S., Addington, J., Cannon, T., et al (2010) Treatment history in the psychosis prodrome: characteristics of the North American Prodrome Longitudinal Study Cohort. Early Intervention in Psychiatry, 4, 220-226.

Das-Munshi, J., Goldberg, D. \& Bebbington, P. E. (2008) Public health significance of mixed anxiety and depression: beyond current classification. British Journal of Psychiatry, 192, 171-177.

Häfner, H., Maurer, K., Löffler, W., et al (1998) The ABC Schizophrenia Study: a preliminary overview of the results. Social Psychiatry and Psychiatric Epidemiology, 33, 380-386.

Judd, L. L., Akiskal, H. S., Schettler, P. J., et al (2005) Psychosocial disability in the course of bipolar I and II disorders: a prospective, comparative, longitudinal study. Archives of General Psychiatry, 62, 1322-1330.

Karsten, J., Hartman, C. A., Ormel, J., et al (2010) Subthreshold depression based on functional impairment better defined by symptom severity than by number of DSM-IV symptoms. Journal of Affective Disorders, 123, 230-237.

Knappe, S., Beesdo, K., Fehm, L., et al (2008) Subthreshold mental disorders in primary care. Psychological Medicine, 58, 72-75.

Lewinsohn, P. M., Shankman, S. A., Gau, J. M., et al (2004) The prevalence and comorbidity of subthreshold psychiatric conditions. Psychological Medicine, 34, 613-622.

Löffler, W. \& Häfner H. (2000) Long prodromal phase in schizophrenia. By recognizing it, the prognosis of the patient can be significantly improved. MMW Fortschritte der Medizin, 142, 26-29.

Mahmoud, S. (1997) Eating Disorders in Egyptian Adolescents. MD thesis in psychiatry, Okasha Institute of Psychiatry, Ain Shams University, Cairo, Egypt.

Merikangas, K. R., Akiskal, H. S., Angst, J., et al (2007) Lifetime and 12 months prevalence of bipolar spectrum disorder in the National Comorbidity Survey replication. Archives of General Psychiatry, 64, $542-543$. 
Okasha, A. (2008) Would the use of dimensions instead of categories remove problems related to subthreshold disorders? European Archives of Psychiatry and Clinical Neuroscience, 259, 129-133.

Regier, D. A., Narrow, W. E., Kuhl, E. A., et al (2009) The conceptual development of DSM-V. American Journal of Psychiatry, 166, 645-650.
Shankman, S. A., Klein, D. N., Lewinsohn, P. M., et al (2008) Family study of subthreshold psychopathology in a community sample. Psychological Medicine, 38, 187-198.

Zlotnik, C., Franklin, C. L. \& Zimmerman, M. (2002) Does subthreshold posttraumatic stress disorder have any clinical significance? Comprehensive Psychiatry, 43, 413-419.

\title{
Children caught up in conflict
}

\author{
David Skuse
}

Behavioural and Brain Sciences Unit, Institute of Child Health, London, UK, email dskuse@ich.ucl.ac.uk

W e present our theme in this issue with an emphasis on the Middle East. Unrest between Israel and Palestine has a long history. Even when there is no overt aggression by either side, decades of conflict may have engendered cognitive distortions and emotional vulnerability.

Danny Brom and colleagues emphasise that there is evidence that even infants and pre-school children in Israel have suffered the consequences of trauma, or anticipated trauma. Their reactions look very much like the post-traumatic stress disorder (PTSD) we see after exposure to a variety of abusive experiences. Up to one-third of children who have been living in areas that are subject to missile attacks in Israel show some symptoms of PTSD. It seems that the duration of exposure to threats of potential violence is a particularly important variable. Similar patterns of disturbance have been observed in older children and adolescents. Interventions based within schools, from early childhood through to adolescence, have been shown to have measurable benefits.

In the Palestinian territory of Gaza, Abdel Aziz Thabet and colleagues describe the evidence for psychiatric disorders following exposure to war during middle childhood and adolescence. The Gaza territory has suffered major structural damage to domestic property in recent years as a consequence of Israeli incursions. The authors were particularly interested in the possibility that components of attention-deficit hyperactivity disorder (as well as PTSD) could be related to exposure to such events. Using a variety of standardised instruments, the team surveyed a representative sample of children from cities, villages and refugee camps. Over a third were found to have experienced at least some PTSD symptoms.

In the third of our thematic papers on the longer-term impact of conflict on children, we revisit the Rwandan genocide. Ian Palmer and Nsanzumuhire Firmin describe the outcomes for children who had been caught up in the terrible events of 1994. They are now in early adulthood. The nature and scale of the depraved behaviour that occurred throughout Rwandan society was probably without precedent in modern times, in terms of both the proportion of the population affected and the involvement of children themselves as perpetrators as well as victims of the violence. Attempts at restorative justice have seemingly not proved very effective in this, Africa's most densely populated country. Half the population was born after the conflict; many children were the product of rape. Many more have HIV/AIDS. Attempts are being made to establish an effective mental health service in Rwanda, but progress is slow.

\section{President's Medals}

During its 2011 International Congress, the College celebrated the achievements and contributions to mental health from people in all walks of life by presenting them with President's Medals. There were nine medal winners this year:

\footnotetext{
- Dr Martin Deahl

- Dr Andrew McCulloch

- Ms Trisha Goddard

- Dr Peter Hughes

- Dr E. S. Krishnamoorthy

- Norman Lamb MP

- Emma Thompson

- Dr Peter Carter

- HE Kjell Magne Bondevik
}

Further information about these medal winners is available on the College website (http://www.rcpsych.ac.uk). 